\title{
Motivación e incentivos laborales en las normas de Gestión de Recursos Humanos del Sector Público
}

\author{
Motivation and work incentives in standards of human \\ resource management in the public sector
}

\section{RESUMEN}

Ante la constatación de la inexistencia de programas de motivación e incentivos laborales en trabajadores del sector público, la investigación tuvo como objetivos determinar la forma en que la normatividad sobre gestión del potencial humano toma en cuenta dichos factores que consideramos de primer orden para lograr la eficacia en el desempeño de la función pública. La investigación abarca dos períodos: el primero comprende desde el año 1884 al 2008 y, el segundo desde el 2008 al 2015, referido este último a la creación de la Autoridad Nacional del Servicio Civil (SERVIR).

Palabras claves: Motivación; incentivos; recursos humanos.

\section{ABSTRACT}

Given the finding of lack of motivation programs and work incentives in public sector workers, the study aims to determine how the regulations on human potential management takes into account such factors we consider first order for effectiveness in the performance of public functions. The research covers two periods: the first comprises since 1884 to 2008 and the second from 2008 to 2015, the latter referred to the creation of the National Civil Service Authority (SERVIR).

Keywords: Motivation; incentives; human resources.

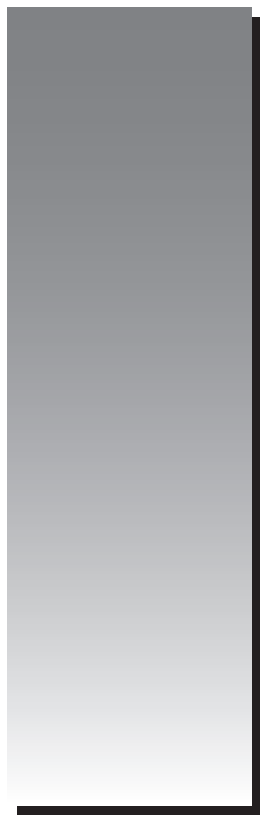

Luis Alonso Castro Pérez ${ }^{1}$

lalonsocastrop@yahoo.es

Félix Armando Rivera León ${ }^{2}$

felix_arl@hotmail.com

Giovanna Jackeline Serna Silva ${ }^{3}$

gia.silvass@gmail.com Universidad Nacional Mayor de San Marcos, Facultad de Ciencias Administrativas

1 Magíster, Docente Asociado T.C. de la Facultad de Ciencias Administrativas de la Universidad Nacional Mayor de San Marcos. Asesor independiente en Gestión Empresarial.

2 Doctor, Docente Principal de la Facultad de Ciencias Administrativas de la Universidad Nacional Mayor de San Marcos. Investigador, Asesor y Ex-Funcionario del sector Público.

3 Doctora, Docente Contratada T.P. de la Facultad de Ciencias Administrativas de la Universidad Nacional Mayor de San Marcos.

(C) Los autores. Este artículo es publicado por Gestión en el Tercer Milenio de la Facultad de Ciencias Administrativas de la Universidad Nacional Mayor de San Marcos. Este es un artículo de acceso abierto, distribuido bajo los términos de la licencia Creative Commons Atribucion - No Comercia_Compartir Igual 4.0 Internacional. (http://creativecommons.org/licenses/by-nc-sa/4.0/) que permite el uso no comercial, distribución y reproducción en cualquier medio, siempre que la obra original sea debidamente citada. 


\section{INTRODUCCIÓN}

La función pública siempre ha estado en el ojo de la tormenta atribuyéndosele burocratismo, ineficiencia y maltrato a los ciudadanos, razones por las cuales los trabajadores públicos son objeto de las peores calificaciones.

Desde la perspectiva de la mirada del sector privado, resulta impensable que una organización que aplica las Ciencias Administrativas a su gestión sea merecedora de tales calificativos.

Lamentablemente, el Estado ha mostrado sostenidamente escasa preocupación por el desarrollo de sus recursos humanos -que en adelante llamaremos potencial humano (por considerarlo más adecuado)-, posiblemente por razones presupuestales, perdiendo de vista la importancia que la función pública cumple en la vida nacional y en el cumplimiento de los fines estatales respecto al bienestar general.

Creemos que una de las razones por las cuales no existen programas de motivación e incentivos laborales, puede tener relación con la equivocada concepción de "motivación" e "incentivos", los cuales son asociados, casi siempre, a lo pecuniario, error al que no escapa el propio Estado. Desde el punto de vista de los trabajadores, ello, podría explicarse por las bajas remuneraciones que perciben; pero que resulta inexcusable para el Estado.

\section{El problema de investigación}

El problema de investigación está referido a determinar ¿En qué medida las normas de gestión del potencial humano del Estado, comprenden acciones de motivación y de incentivos laborales? y la forma en que estos se implementarían.

Las normas de gestión del potencial humano constituyen medidas necesarias ex ante, es decir, que justifican su existencia por la necesidad de regular la incorporación de personas a la función pública. Sin embargo, en la práctica existe escasa relación entre las nociones "servidor público" y "función pública”, entendiéndose al primero como un simple operador de actividades reguladas por ley y a la segunda como las obligaciones que el primero debe cumplir.

Debido a esa dinámica, el rol de los servidores públicos se ha convertido en una rutina monótona, sin un fin plenamente identificado; en consecuencia, alejado de su principal objetivo: servir al país para el logro del bienestar general. Este defecto ha sido asumido y reforzado por los responsables de la gestión pública, a quienes incluso, les conviene tal situación, porque permite mantener las condiciones laborales actuales.

Más allá de los pareceres antes expuestos, conviene determinar si efectivamente, el Estado ignora conscientemente la necesidad de ofrecer motivación e incentivos laborales a sus trabajadores, por lo que es necesario explorar en las normas regulatorias de la relación laboral entre ambos.

\section{Relación del estudio con otros similares}

Nuestra investigación no está vinculada a ningún otro estudio en curso.

\section{Justificación}

Para que las instituciones sean eficaces, requieren, contar con personal debidamente identificado y motivado, características que se desarrollan a partir de una adecuada gestión de potencial humano. Tales atributos, sin embargo, no forman parte de la gestión que lleva a cabo el Estado respecto a sus servidores públicos, siendo una muestra de ello que la Dirección de Políticas de la Autoridad Nacional del Servicio Civil (SERVIR), no ha sido capaz de dictar una sola política en ocho (8) años de existencia.

Cuando los programas de motivación e incentivos están ausentes en una organización, difícilmente se logra articular el desarrollo de las tareas con las metas y los objetivos organizacionales, cuestión que, hasta el momento, como demostraremos, no es entendida por el Estado.

\section{Limitaciones}

En cuanto a las limitaciones, no hemos encontrado ninguna respecto al acceso a las normas analizadas, porque estas son de dominio público.

\section{Gestión de recursos humanos en el sec- tor público}

Dice Klingner (2003):

La administración de personal público se ha definido desde cuatro puntos de vista. El primero es el 
de las funciones que se necesitan para administrar los recursos humanos en las agencias públicas. El segundo es el del proceso a través del cual se asignan los puestos públicos. El tercero es el de la interacción de los valores sociales fundamentales entre sí, dichos valores se encuentran muchas veces en conflicto al determinar quiénes consiguen puestos públicos y en qué forma se asignan dichos puestos. El último punto de vista se refiere a los sistemas de personal -- las leyes, las reglas, las organizaciones y los procedimientos que se utilizan para expresar estos valores abstractos al cumplir las funciones de la administración de personal.

Los vertiginosos cambios en la ciencia, tecnología, economía y política mundiales, tienen actualmente una fuerte influencia en la gestión del potencial humano público, desde el momento en que la concepción de Estado, ha ido cambiando también por efectos de la globalización.

\begin{abstract}
Según Cassagne (2002):
A partir de la segunda mitad de este siglo ha venido desenvolviéndose, en diversos países del mundo, uno de los procesos de transformación de mayor magnitud acerca del papel que corresponde asumir al Estado, Su origen, particularmente en Europa Occidental, ha respondido más a las exigencias de la realidad que a las ideologías y ha sido apoyado, en gran medida, por el derecho de las comunidades europeas que, en virtud del principio de supremacía, prevalece sobre las normas de las constituciones formales de los Estados que la integran.
\end{abstract}

Desde ese punto de vista, el Estado se percibe a sí mismo como un elemento que propicia el cambio, alentando al sector privado a invertir y generar mayor riqueza y empleo, para cuyo efecto cambia su estructura y funciones, moderniza sus procedimientos, capacita a su potencial humano, elimina las barreras burocráticas de acceso al mercado y promueve la inversión para lograr ampliar el mercado laboral.

Los servidores públicos juegan un rol trascendente y la gestión del potencial humano de la Administración Pública se convierte en una prioridad, lo cual requiere un cuerpo normativo sólido que debe contemplar claramente la forma en que se establece el vínculo entre los servidores públicos y el Estado, como también las políticas de gestión y las líneas de carrera.

Para Klingner, los cambios mundiales tienen como consecuencias en la administración de recursos humanos:
- "El cambio de la administración de posiciones a la administración de trabajo y de empleados.

- La diferenciación precisa entre empleos básicos y contingentes.

- Los desafíos del sistema tradicional de servicio civil.

- El crecimiento de las entidades y los mecanismos alternativos."

Agrega que dichos cambios afectan al papel del administrador de personal público de la siguiente manera:

- "Las consecuencias de dichos desafíos para el desempeño de los empleados y el liderazgo.

- La necesidad de unir los puntos de vista políticos y administrativos.

- El papel complejo y cambiante del administrador de personal público."

En tal sentido, la gestión de potencial humano cobra cada vez mayor complejidad por la cantidad de variables a ser tomadas en cuenta y por la permanente vigilancia que es necesario hacer del entorno para atender a las también crecientes necesidades sociales. Quiere decir, que la gestión de potencial humano cobra mayor impulso y dinamismo, así como gran importancia en el fortalecimiento del Estado.

Refiriéndose a la actuación de la Administración, afirma Cassagne (2002), que ésta:

(...) se realiza a través de actos o hechos que traducen el ejercicio o realización de actividades de diversa índole en punto a su esencia o sustancia material. Por su propia naturaleza, la Administración Pública desarrolla una actividad material y objetivamente administrativa, de alcance individual y concreta, tendiente a satisfacer, en forma inmediata, las necesidades de bien común o de interés público, cuya concreción resulta indispensable en toda comunidad jurídicamente organizada. Tal es su actividad predominante.

Un aspecto de suma relevancia en dicho fortalecimiento lo constituye la identificación institucional y esto sólo es posible estableciendo una carrera administrativa que, además de fijar deberes, obligaciones y responsabilidades, 
ofrezca a los agentes públicos la posibilidad de realizarse laboralmente. De otra manera, los servidores públicos adoptarán un papel conformista que, a la postre, mantendrá la ineficiencia estatal.

Más allá de los considerandos que apelan de manera superflua a argumentos de supuesta modernidad de la Administración pública, lo que se pretende ocultar es la evidente intención de convertir el empleo público en un empleo precario, sin tomar en consideración el riesgo que ello significa. En efecto, por la forma en que se está implementado la política de gestión de potencial humano, es de esperarse que llegue el momento en que el sistema colapse. En ese orden de ideas, no debemos perder de vista que los servidores públicos son mandatarios, es decir, que actúan en representación del Estado y que, por lo tanto, la práctica de los más altos valores éticos debe caracterizar a la gestión pública.

Desde nuestro punto de vista, la gestión del potencial humano, reúne requisitos que deben cumplirse previamente, como el establecimiento de los objetivos y funciones que debe cumplir el Estado en el nuevo escenario mundial con la finalidad de llevar a cabo la reforma estructural del Estado, para, luego, determinar la calidad y cantidad de servidores que se necesitan de acuerdo a dichas funciones, de modo que la legis- lación sobre servidores públicos responda adecuadamente a las necesidades sociales (Figura 1).

En el Fórum, organizado por el Congreso de la República (2005), Pierina Pollarolo, sostuvo:

(...) la ausencia de orientación a resultados en los trabajadores del Estado. Actualmente la retribución a los trabajadores no guarda ninguna relación con que éstos alcancen los objetivos y metas que les corresponden según el plan de trabajo de su entidad. Así, no se puede premiar a quienes brindan mejores servicios de educación o llamar la atención a quienes no cumplen con brindar servicios de salud con calidad y oportunidad para la población más necesitada.

\section{La Carta Iberoamericana de Calidad en la Función Pública (2003)}

El Preámbulo de la Carta Iberoamericana de Calidad en la Función Pública (2003), señala que, de conformidad con la Declaración de Santo Domingo, aprobada por la IV Conferencia Iberoamericana de Ministros de Administración Pública y Reforma del Estado:

(...) el Estado constituye la máxima instancia de articulación de relaciones sociales. Desde el punto de vista de la gobernabilidad, el progreso económico y la reducción de la desigualdad social, el papel del Estado en las sociedades contemporáneas, y en particular en el área iberoamericana, es fundamental para el logro de niveles crecientes de bienestar colectivo.

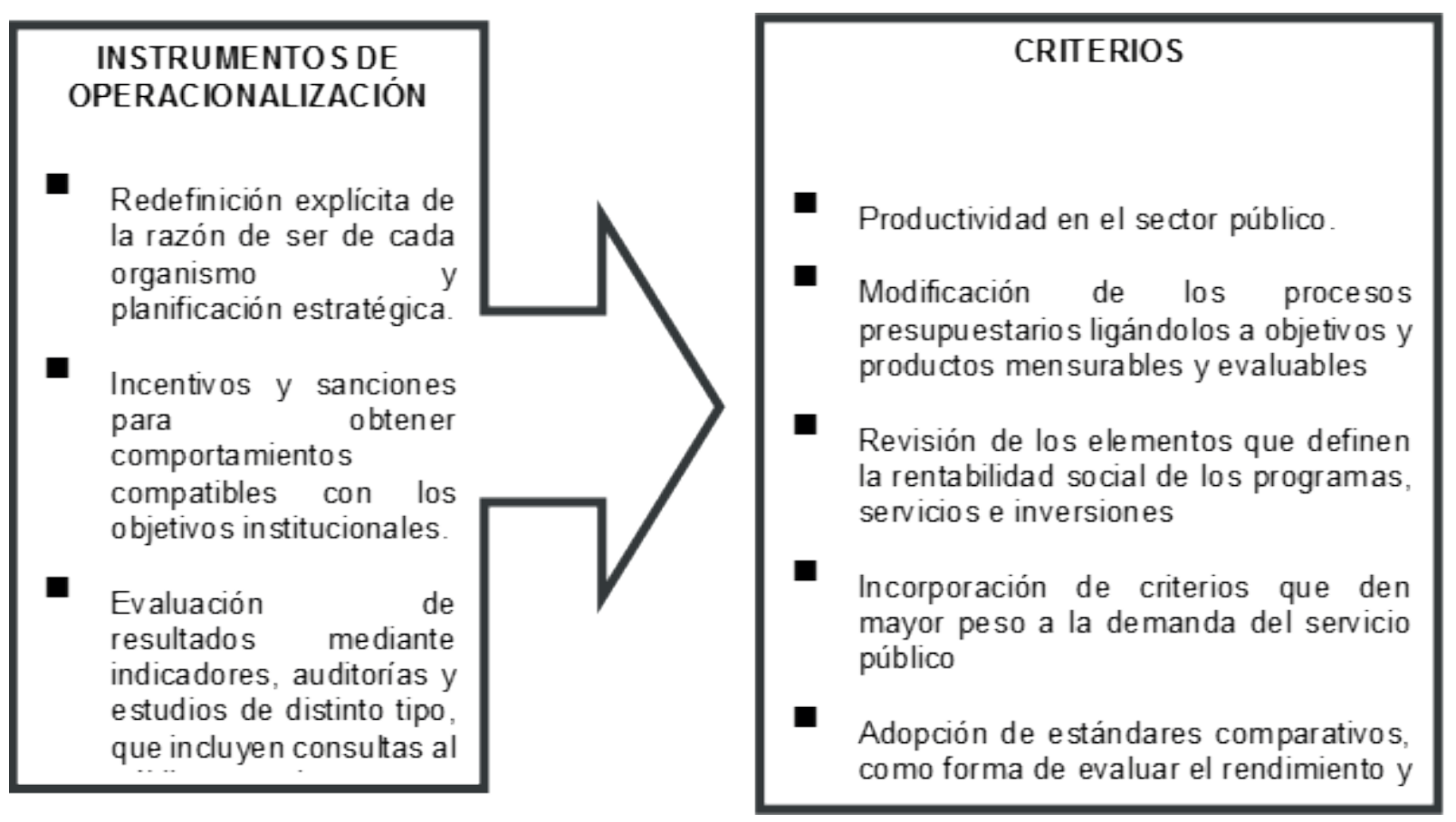

Figura 1. Gestión de recursos humanos en el sector público 
En ese sentido, considera que la mejora del Estado, debe basarse en la profesionalización de la función pública, entendiendo que dicha profesionalización debe traducirse en:

(...) posesión por los servidores públicos de una serie de atributos como el mérito, la capacidad, la vocación de servicio, la eficacia en el desempeño de su función, la responsabilidad, la honestidad y la adhesión a los principios y valores de la democracia.

Nótese que este concepto, va más allá de las consideraciones expuestas como condiciones de los empleados públicos en las normas legales de nuestro país, porque apuntan al afianzamiento del Estado Democrático de Derecho.

Asimismo, equipara el concepto de "función pública" al de "servicio civil", excluyendo de ambos conceptos a los cargos de naturaleza política. Sostiene, además, que los sistemas de función pública o servicio civil, con las características antes señaladas, guardan relación con "(...) los niveles de confianza de los ciudadanos en la administración pública, la eficacia gubernamental y la lucha contra la corrupción, y la capacidad de crecimiento económico sustentable de los países", como también “(...) contribuye al fortalecimiento institucional de los países y a la solidez del sistema democrático."

Define la función pública, señalando que:

“(...) está constituida por el conjunto de arreglos institucionales mediante los que se articulan y gestionan el empleo público y las personas que integran éste, en una realidad nacional determinada. Dichos arreglos comprenden normas, escritas o informales, estructuras, pautas culturales, políticas explícitas o implícitas, procesos, prácticas y actividades diversas cuya finalidad es garantizar un manejo adecuado de los recursos humanos, en el marco de una administración pública profesional y eficaz, al servicio del interés general",

Al mismo tiempo que privilegia la importancia del potencial humano como tal, con el añadido de su profesionalización, su capacidad de adaptación al cambio, la garantía de su estabilidad laboral, el comportamiento ético y la asunción de las correspondientes responsabilidades, pero también el protagonismo que debe caracterizar a los responsables de la gestión, añadiéndose la necesidad de establecer canales de comunicación y participación en la gestión, además del establecimiento de políticas para favorecer la igualdad de género, la protección e integración de las minorías, y en general la inclusión y la no discriminación por motivos de género, origen social, etnia, discapacidad u otras causas

Establece como requerimientos de la función pública:

1. La planificación de potencial humano. A través de la cual la organización realiza el estudio de sus necesidades cuantitativas y cualitativas de potencial humano a corto, medio y largo plazo, contrasta las necesidades detectadas con sus capacidades internas, e identifica las acciones que deben emprenderse para cubrir las diferencias.

2. La organización del trabajo. Mediante el uso de instrumentos de gestión de potencial humano que permitan definir las características y la descripción de los puestos de trabajo, así como los requisitos de idoneidad de las personas llamadas a desempeñarlas (perfiles de competencias.

Respecto a la evaluación del rendimiento, señala que ésta permite:

1. "Obtener informaciones necesarias para adoptar decisiones en diferentes áreas de la gestión de las personas (remuneración, promoción, capacitación, disciplina...)

2. Validar políticas y prácticas de gestión de recursos humanos, contrastando y valorando su impacto sobre el comportamiento humano en el trabajo.

3. Orientar el desarrollo de las personas y su crecimiento profesional.

4. Mejorar la motivación y el rendimiento de las personas en el puesto de trabajo."

Las características de la retribución aquí expuestas, guardan la debida coherencia con la calidad del servicio que debe primar en la administración. 


\section{Carta Iberoamericana de Calidad en la Gestión Pública}

La Carta Iberoamericana de Calidad en la Gestión Pública (2008) destaca el rol del Estado en el desarrollo económico, político y social de cualquier país, sugiriendo que en la región, debe enfocar su acción en la atención de tres grandes problemas: la consolidación de la democracia, la necesidad de retomar el crecimiento económico y la reducción de la desigualdad social, garantizando la inclusión social, poniendo como condición para ello la adecuación de su organización y funcionamiento a las nuevas realidades, aprendiendo de los errores y aciertos de las diversas experiencias recientes y adoptando un nuevo modelo de gestión pública que recupere la capacidad de las Administraciones Públicas como instrumentos útiles y efectivos al servicio del bien común o interés general de sus respectivas sociedades.

Como rasgo importante de la Carta, está la mención que hace al derecho de los ciudadanos a una gestión pública de calidad, que a su vez contribuya a su empoderamiento, de manera que puedan exigir la mejora continua de la gestión pública en su propio beneficio, así como participar directamente en ella.

Si bien en nuestro país, se ha ido ampliando cada vez más el nivel de participación ciudadana, no puede afirmarse que se haya logrado una mejora sustancial en la gestión pública, presumiblemente porque la sola participación resulta insuficiente si el mismo Estado no asume acciones claras y coherentes para mejorar sus procedimientos y sus recursos humanos.

Sostiene que "La calidad en la gestión pública constituye una cultura transformadora que impulsa a la Administración Pública a su mejora permanente para satisfacer cabalmente las necesidades y expectativas de la ciudadanía con justicia, equidad, objetividad y eficiencia en el uso de los recursos públicos".

\section{La motivación e incentivos laborales en el sector público}

Existe una diversidad de normas que regulan la gestión del potencial humano del sector público, las cuales contemplan los derechos, deberes y obligaciones de los servidores públicos, además de normas referidas al comportamiento ético, incentivos y sanciones.

\section{Decreto Legislativo № 276, Ley de Bases de la Carrera Administrativa y de Remuneraciones del Sector Públi- co y su Reglamento Decreto Supremo $\mathrm{N}^{\circ}$ 005-90-PCM}

El Decreto Legislativo № 276, Ley de Bases de la Carrera Administrativa y de Remuneraciones del Sector Público, no contempla disposición alguna referida a motivación e incentivos.

El Artículo 10을 del Reglamento de la ley, reconoce el mérito laboral como un elemento vinculado a reconocimiento interpretado como el grado de eficiencia y responsabilidad con que desempeña el servidor las funciones asignadas.

El Artículo 140으 del Reglamento, establece la obligación de las entidades públicas de "...diseñar y establecer políticas para implementar, de modo progresivo, programas de bienestar social e incentivos..." los cuales, están definidos en el Artículo 147º, como sigue:

"Los programas de incentivos atenderán los siguientes aspectos:

a. Reconocimiento de acciones excepcionales o de calidad extraordinaria relacionadas directamente o no con las funciones desempeñadas, a saber:

- Agradecimiento o felicitación escrita.

- Diploma y medalla al mérito.

- La Orden del Servicio Civil, en sus diferentes grados.

b. Otorgamiento de becas y préstamos por estudios o capacitación.

c. Programas de turismo interno anual para los servidores de carrera distinguidos, de acuerdo a la disponibilidad presupuestal de la entidad.

d. Promoción y publicación de trabajos de interés especial para la entidad y la Administración Pública.

e. Compensación horaria de descanso por trabajo realizado en exceso al de la jorna- 
da laboral, siempre y cuando no pudiera ser remunerada.

f. Subsidio por la prestación de servicios en zonas geográficas de alto riesgo y menor desarrollo de acuerdo a las prioridades que se establezcan en cada región.

g. Otorgamiento de un día de descanso por el onomástico del servidor.

h. Otros que pudieran establecerse por norma expresa.

Asimismo, el Artículo 148으, prescribe que "El desempeño excepcional que origine especial reconocimiento, deberá enmarcarse en las condiciones siguientes:

a. Constituir ejemplo para el conjunto de servidores.

b. Que esté orientado a cultivar valores sociales mencionados en la Constitución del Estado.

c. Que redunde en beneficio de la entidad.

d. Que mejora la imagen de la entidad en la colectividad.

En la práctica, tales incentivos nunca fueron puestos en práctica, porque al haberse dado el Reglamento el año 1990, con el shock económico de 1992, la carrera pública quedó congelada y, con ello, todas las disposiciones referidas a la gestión del potencial humano que significaran gasto público.

\section{Decreto Legislativo $\mathrm{N}^{\circ} 1057$, Régimen Especial de Contratación Adminis- trativa de Servicios y Decreto Supre- mo № 075-2008-PCM, Reglamento del Decreto Legislativo № 1057, que regula el régimen especial de con- tratación administrativa de servicios}

Este régimen ya eliminado, definía el Contrato Administrativo de Servicios en su Artículo 3 , como "...una modalidad especial propia del derecho administrativo y privativa del Estado."

El Decreto Supremo № 075-2008-PCM, Reglamento del Decreto Legislativo № 1057, que regulaba el régimen especial de contratación administrativa de servicios (CAS), se limitaba a establecer el procedimiento para la celebración del contrato, su contenido y los supuestos de suspensión y culminación de la relación laboral.

El Decreto Supremo № 065-2011-PCM, establece modificaciones al Reglamento del Régimen de CAS, tampoco aporta elementos a favor de la motivación e incentivos a los trabajadores contratados, no obstante que, actualmente, su número casi igual al de los servidores permanentes y la mayoría labora desde hace más de veinte años para el Estado.

La Ley $\mathrm{N}^{\circ} 29849$, establece la eliminación progresiva del Régimen Especial del Decreto Legislativo $\mathrm{N}^{\circ} 1057$ y otorga derechos laborales. La presente ley tiene por objetivo establecer la eliminación del Régimen Especial de Contratación Administrativa de Servicios, regulado mediante el Decreto Legislativo 1057. Esta ley entró en vigencia desde el 12 de abril del 2012. Norma autoaplicativa cuya vigencia será inmediata e incondicionada, a larga podría traer beneficios a los trabajadores empleados bajo este régimen.

La Ley № 30057, Ley del Servicio Civil, actualmente en proceso de implementación para las entidades que se han adecuado a ella, precisa en su Artículo 24, referido a los factores de evaluación del desempeño que esta "... se realiza tomando en cuenta, principalmente, factores o metas individuales relacionadas a la función que desempeña el servidor. Adicionalmente, se pueden tomar en cuenta factores grupales cuando se hubiera establecido oficialmente indicadores de gestión para la entidad y sus unidades orgánica", es decir, se valora un elemento subjetivo, no necesariamente promovido o impulsado institucionalmente.

A diferencia de las normas antes comentadas, la Ley introduce el término "Compensación" para referirse a la remuneración, señalando en su Artículo 28, que esta "...es el conjunto de ingresos y beneficios que la entidad destina al servidor civil para retribuir la prestación de sus servicios a la entidad de acuerdo al puesto que ocupa" siendo su objetivo "...captar, mantener y desarrollar un cuerpo de servidores efectivo que contribuya con el cumplimiento de los objetivos institucionales." 
El Artículo 29, además de contemplar la compensación o remuneración, establece una compensación no económica, “...constituida por los beneficios otorgados para motivar y elevar la competitividad de los servidores civiles. Estos beneficios no son de libre disposición del servidor".

Más adelante, el Artículo 30, establece como principios de la compensación:

a. Competitividad: El sistema de compensaciones busca atraer y retener personal idóneo en el Servicio Civil peruano.

b. Equidad: Al trabajo desempeñado en puestos similares, pero en condiciones diferentes de exigencia, responsabilidad o complejidad le corresponde diferente compensación económica y al trabajo desempeñado en puestos y condiciones similares le corresponde similar compensación económica.

c. Consistencia interna: Las compensaciones dentro de la misma entidad guardan relación con las condiciones de exigencia, responsabilidad y complejidad del puesto.

d. Consistencia intergubernamental: Las compensaciones de puestos similares, entre las entidades de la administración pública son comparables entre sí. Esta regla se aplica teniendo en cuenta el nivel de responsabilidad y competencias de la entidad.

Las disposiciones contenidas en los artículos precedentes, nos hacen ver una clara diferenciación de orden discriminatorio entre entidades y servidores, dejando librado el otorgamiento de las compensaciones a la voluntad del gobierno decidir qué entidades son las que reúnen condiciones de exigencia, responsabilidad o complejidad que justifiquen una mayor compensación, tal como ocurre actualmente respecto al incentivo al Comité de Administración del Fondo de Asistencia y Estímulo (CAFAE), que ha marcado una enorme diferencia entre servidores del Ministerio de Economía y Finanzas (MEF) cuyos montos pueden superar los S/. 10,000.00 frente a servidores de otras entidades que reciben S/. 450.00.

Queda también en discusión los criterios que asumirán quienes determinen el mérito individual a fin de conceder las compensaciones no económicas.

\section{MÉTODOS}

En la presente investigación, por ser de tipo teórico, no hemos utilizado sujetos como muestra, siendo nuestro material de estudio el conjunto de normas legales vinculados a la función pública.

\section{RESULTADOS Y DISCUSIÓN}

Como producto del estudio, se ha encontrado:

1. En la Administración Pública peruana, existe una diversidad de normas regulatorias de la relación entre el Estado y sus servidores, las cuales se refieren fundamentalmente a definir la relación, establecer los mecanismos de incorporación, establecer los derechos, deberes, y sanciones por la comisión de faltas.

El hecho que se mantengan vigentes diversas normas crea, en principio una diversidad de servidores con diferentes derechos, pero las mismas obligaciones, polarizando la relación entre ambas y dando paso al conflicto, como ocurre entre servidores permanentes y contratados. Este hecho afecta también la motivación, porque los trabajadores permanentes gozan de estabilidad laboral y perciben beneficios superiores a los contratados, sintiéndose estos marginados, además de encontrarse en permanente estado de tensión por la posibilidad de no renovación de sus contratos, que se produce cada tres meses.

2. La ausencia de políticas de potencial humano, no obstante existir una Autoridad Nacional del Servicio Civil, con una Dirección de Políticas de Recursos Humanos.

El Estado, a través del ente rector de gestión del potencial humano, elude su responsabilidad, generando un grave 
daño al país. Resulta contraproducente que quienes tienen la responsabilidad de lograr la mayor eficiencia en la función pública, sean los responsables de su debacle. Lamentablemente, SERVIR, ha sido incapaz de realizar planteamientos de coherentes para un mejor servicio público.

3. El vacío legal respecto a la motivación y otorgamiento de incentivos como parte de programas orientados a mejorar la eficiencia y eficacia estatal.

A pesar de las deficiencias mostradas por SERVIR, esta entidad obliga a las entidades del Estado a formular el Plan de Desarrollo de Personas, documento que debiera permitir llenar el vacío legal y contener, obligatoriamente, los programas de motivación e incentivos de la entidad.

4. La regulación de la nueva carrera administrativa, no obstante ser de corte privatista, ignora los principios de la moderna administración, manteniéndose en el viejo molde de exigir esfuerzo al trabajador a cambio de compensaciones que no satisfacen sus necesidades más elementales, aduciéndose, siempre, como justificación, las limitaciones presupuestales.

5. Los incentivos ofrecidos en la Ley del Servicio Civil se ofrecen casi como una dádiva a aquellos que se "esfuercen más", sin que su implementación responda a un programa definido respaldado por una política.

En cuanto a la generalización de resultados, estos no encuentran límite, puesto que son aplicables a todas las entidades del Estado.

\section{CONCLUSIONES}

El Estado, no obstante tener una finalidad claramente diferenciada de las organizaciones de orden económico a cargo de los agentes económicos, posee características muy similares que lo hacen perfectamente capaz de adaptar las herramientas de la moderna administración científica al desarrollo de sus actividades.

En ese sentido, incorporar los conocimientos de la administración de potencial humano a la administración pública, resulta una cuestión crucial, tanto desde el punto de vista de la capital importancia del papel del Estado frente a la sociedad, como del significado que tiene para la individualidad de los servidores públicos.

Por tal motivo resulta científicamente inexplicable que las normas que rigen la relación entre el estado y sus servidores adolezcan de un vacío tan importante, cuestión que, a nuestro modo de ver, tiene un peso gravitante en la eficacia estatal y cuyos resultados son tan antiguos como la propia república. Además de las razones presupuestales que señalamos como razón para no desarrollar programas de motivación e incentivos, creemos que el estado no puede liberarse, aún, de las taras del pasado, manifestadas en su forma de gestión.

Por otro lado, percibimos una escasa capacidad para entender cabalmente los conceptos de "motivación" e "incentivos", los cuales no terminan de ser asumidos plenamente en las disposiciones gubernamentales. En efecto, el término "motivación", apenas si es mencionado con timidez en algún momento, pero sin darle el peso específico que la gestión requiere, mientras que los "incentivos" son distorsionados en sus objetivos, porque son colocados como "la zanahoria del burro de Buridán".

Otro hecho destacable en la normatividad pública es el exceso de "deberes", "obligaciones" y "responsabilidades" con que carga al servidor público, como si fuera el gran culpable de los males de la administración, con lo cual se crea una atmósfera amenazante, demostrándose la existencia del enfoque de la Teoría X de la administración y un estilo de liderazgo autoritario. Con esto no queremos decir que no exista exigencia de obligaciones, pero lo que ocurre con la Administración Pública es que es más lo que quita que lo que da.

Desde nuestro de punto de vista, la mejora continua del potencial humano del Estado, exige un cambio de visión de los responsables de la gestión pública, orientada al desarrollo de un Estado moderno, como también acerca de la trascendencia de contar con servidores adecuadamente preparados, tanto técnica como personalmente para afrontar sus responsabilidades ante la sociedad. 


\section{REFERENCIAS BIBLIOGRÁFICAS}

Carta Iberoamericana de Calidad en la Gestión Pública, Aprobada por la X Conferencia Iberoamericana de Ministros de Administración Pública y Reforma del Estado. San Salvador, El Salvador, 26 y 27 de junio de 2008. Adoptada por la XVIII Cumbre Iberoamericana de Jefes de Estado y de Gobierno. San Salvador, El Salvador, del 29 al 31 de octubre de 2008.

Carta Iberoamericana de la Función Pública, Aprobada por la V Conferencia Iberoamericana de Ministros de Administración Pública y Reforma del Estado. Santa Cruz de la Sierra, Bolivia, 26-27 de junio de 2003. Respaldada por la XIII Cumbre Iberoamericana de Jefes de Estado y de Gobierno (Resolución № 11 de la "Declaración de Santa Cruz de la Sierra") Bolivia, 14-15 de noviembre de 2003.

Carta Iberoamericana de Participación Ciudadana en la Gestión Pública, Aprobada por la XI Conferencia Iberoamericana de Ministros de Administración Pública y Reforma del Estado. Lisboa, Portugal, 25 y 26 de junio de 2009. Adoptada por la XIX Cumbre Iberoamericana de Jefes de Estado y de Gobierno Estoril, Portugal, 30 de noviembre y $1^{\circ}$ de diciembre de 2009 .

Cassagne, Juan Carlos: Derecho Administrativo. TOMO I. Séptima edición actualizada. Lexis Nexis, Abeledo-Perrot. Buenos Aires. 2002. Pág. 58.

Cassagne, Juan Carlos: Derecho Administrativo. TOMO II. Séptima edición actualizada. Lexis Nexis, Abeledo-Perrot. Buenos Aires. 2002. Pág. 11.
Congreso de la República - Programa Pro Descentralización: Fórum "En el Camino de la Reforma del Empleo Público” Octubre, 2005. Lima-Perú.

Decreto Legislativo $N^{\circ}$ 1057, Régimen Especial de Contratación Administrativa de Servicios.

Decreto Legislativo № 276, Ley de Bases de la Carrera Administrativa y de Remuneraciones del Sector Público.

Decreto Supremo N 005-90-PCM, Reglamento del Decreto Legislativo № 276.

Decreto Supremo № 075-2008-PCM, Reglamento del Decreto Legislativo № 1057.

FUENTES NAVARRO, Silvia María: (2012) Satisfacción laboral y su influencia en la productividad (Estudio realizado en la delegación de recursos humanos del organismo judicial en la ciudad de Quetzaltenango) Tesis para optar el Título de Psicóloga Industrial/Organizacional. Facultad de Humanidades, Universidad Rafael Landívar. Guatemala.

KLINGNER, Donald: La administración de recursos humanos y la modernización del Estado. VIII Congreso Internacional del CLAD sobre la Reforma del Estado y de la Administración Pública, Panamá, 28-31 oct. 2003.

Ley $N^{\circ} 29849$, Ley que establece la eliminación progresiva del Régimen Especial del Decreto Legislativo $\mathrm{N}^{\circ} 1057 \mathrm{y}$ otorga derechos laborales.

Ley N³0057, Ley del Servicio Civil.

MATACHI, Atsushi (2006). Capacity Building Framework UNESCO-IICBA. UNESCO - International Institute for Capacity Building in Africa. 